\title{
Isolation and characterization of a motility-defective mutant of Euglena gracilis
}

\author{
Shuki Muramatsu ${ }^{1,2}$, Kohei Atsuji ${ }^{3,4}$, Koji Yamada ${ }^{\text {Corresp., 3,4 }}{ }^{\text {, Kazunari Ozasa }}{ }^{4}$, Hideyuki Suzuki ${ }^{3}$ Takuto Takeuchi $^{3}$,

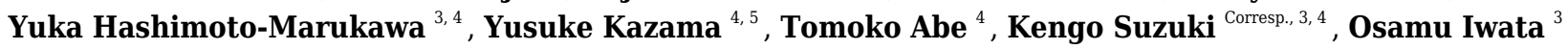 \\ ${ }^{1}$ Showa Women's University, Tokyo, Japan \\ 2 Euglena Co., Ltd., Tokyo, Japan \\ 3 euglena Co., Ltd., Tokyo, Japan \\ 4 RIKEN, Wako, Japan \\ 5 Fukui Prefectural University, Fukui, Japan \\ Corresponding Authors: Koji Yamada, Kengo Suzuki \\ Email address: yamada@euglena.jp, suzuki@euglena.jp
}

Euglena gracilis is a green photosynthetic microalga that swims using its flagellum. This species has been used as a model organism for over half a century to study its metabolism and the mechanisms of its behavior. The development of mass-cultivation technology has led to $E$. gracilis application as a feedstock in various products such as foods. Therefore, breeding of $E$. gracilis has been attempted to improve the productivity of this feedstock for potential industrial applications. For this purpose, a characteristic that preserves the microalgal energy e.g., reduces motility, should be added to the cultivars. The objective of this study was to verify our hypothesis that $E$. gracilis locomotion-defective mutants are suitable for industrial applications because they save the energy required for locomotion. To test this hypothesis, we screened for E. gracilis mutants from Fe-ion-irradiated cell suspensions and established a mutant strain, $\mathrm{M}_{3}^{-} \mathrm{ZFeL}$, which shows defects in flagellum formation and locomotion. The mutant strain exhibits a growth rate comparable to that of the wild type when cultured under autotrophic conditions, but had a slightly slower growth under heterotrophic conditions. It also stores 1.6 times the amount of paramylon, a crystal of $\beta$-1,3-glucan, under autotrophic culture conditions, and shows a faster sedimentation compared with that of the wild type, because of the deficiency in mobility and probably the high amount of paramylon accumulation. Such characteristics make E. gracilis mutant cells suitable for cost-effective mass cultivation and harvesting. 


\section{Isolation and characterization of a motility-defective} 3 mutant of Euglena gracilis

4

5

6 Shuki Muramatsu ${ }^{1, \dagger}$, Kohei Atsuji ${ }^{1,2}$, Koji Yamada ${ }^{1,2}$, Kazunari Ozasa ${ }^{3}$, Hideyuki Suzuki ${ }^{1}$,

7 Takuto Takeuchi ${ }^{1}$, Yuka Hashimoto-Marukawa ${ }^{1,2}$, Yusuke Kazama ${ }^{4, \ddagger}$, Tomoko Abe ${ }^{4}$, Kengo

8 Suzuki $^{1,2}$, Osamu Iwata ${ }^{1,2}$

$9 \quad{ }^{1}$ R\&D Department, euglena Co., Ltd., Minatot-ku, Tokyo, Japan

$10{ }^{2}$ Microalgae Production Control Technology Laboratory, RIKEN, Wako, Saitama, Japan

$11{ }^{3}$ Bioengineering Laboratory, RIKEN, Wako, Saitama, Japan

12 13

14

15

${ }^{4}$ Nishina Center for Accelerator-based Science, RIKEN, Wako, Saitama, Japan

$\dagger$ Present affiliation:

Faculty of Life and Environmental Sciences, Showa Women's University, Setagauya-ku Tokyo, Japan

$\$$ Present affiliation:

Department of Bioscience and Biotechnology, Fukui Prefectural University, Yoshida-gun, Fukui, Japan

Corresponding Author:

Koji Yamada ${ }^{1,2}$

5-29-11 Shiba, Minato-ku, Tokyo 108-0014, Japan

Email address: yamada@euglena.jp

Kengo Suzuki ${ }^{1,2}$

5-29-11 Shiba, Minato-ku, Tokyo 108-0014, Japan

Email address: suzuki@euglena.jp

Osamu Iwata 1,2

5-29-11 Shiba, Minato-ku, Tokyo 108-0014, Japan

Email address: iwata@euglena.jp 


\section{Abstract}

39 Euglena gracilis is a green photosynthetic microalga that swims using its flagellum. This species

40 has been used as a model organism for over half a century to study its metabolism and the mechanisms of its behavior. The development of mass-cultivation technology has led to $E$. gracilis application as a feedstock in various products such as foods. Therefore, breeding of $E$. applications. For this purpose, a characteristic that preserves the microalgal energy e.g., reduces motility, should be added to the cultivars. The objective of this study was to verify our hypothesis that $E$. gracilis locomotion-defective mutants are suitable for industrial applications because they save the energy required for locomotion. To test this hypothesis, we screened for $E$. gracilis mutants from Fe-ion-irradiated cell suspensions and established a mutant strain, $\mathrm{M}^{-}$ ${ }_{3} \mathrm{ZFeL}$, which shows defects in flagellum formation and locomotion. The mutant strain exhibits a growth rate comparable to that of the wild type when cultured under autotrophic conditions, but had a slightly slower growth under heterotrophic conditions. It also stores 1.6 times the amount of paramylon, a crystal of $\beta$-1,3-glucan, under autotrophic culture conditions, and shows a faster sedimentation compared with that of the wild type, because of the deficiency in mobility and probably the high amount of paramylon accumulation. Such characteristics make E. gracilis mutant cells suitable for cost-effective mass cultivation and harvesting.

\section{Introduction}

The locomotion of organisms is directly linked to characteristic evolutionary survival competitions, such as acquiring food, finding suitable environments, and escaping from predators (Domenici et al. 2007). Locomotion in response to environmental stimuli is defined as "taxis," including chemotaxis, gravitaxis, and phototaxis, which respectively refer to locomotion toward chemicals, gravity, and light, respectively (Dusenbery 2009). Such taxes are essential for organisms to survive in natural environments and are regulated by complex mechanisms (Webre et al. 2003; Okita 2005; Roberts 2006; Jékely et al. 2008; Roberts 2010; Sourjik and Wingreen 2012). The means of locomotion depend on the organism size and are optimized through evolution (Jahn and Bovee 1965; Dusenbery 1997). Many of the motile unicellular microorganisms in the hydrosphere use their flagella to swim (Miyata et al. 2020).

Among the microalgae, Chlamydomonas reinhardtii has been widely used as a model organism to study the molecular mechanisms underlying the swimming of phytoplankton cells using their flagella. C. reinhardtii is used as a model microalga because its genetic methodology has been established (Harris 2001). Using their gravitaxis and phototaxis, strategies to screen non-motile $C$. reinhardtii mutants were developed (Kamiya 1991). The acquisition of these mutants and their analysis elucidated the molecular mechanisms used for swimming; e.g. the interaction between microtubule and dynein motor protein drives the motion of the flagella (Kamiya and Okamoto 1985; Kamiya et al. 1991; Kamiya 1995). The photosynthetic flagellate 
77 species of the genus Euglena also use their flagella to swim, but they can be shed in response to 78 chemical or mechanical stimuli (Bovee 1982). In contrast to Chlamydomonas cells, which cannot

79

80

81

82

83

84

85

86

87

88

89

90

91

92

93

94

95

96

97

98

99

100

101

102

103

104

105

106

107

108

109

110

111

112

113

114

115

116

Euglena gracilis is extensively used as a model organism to study the mechanisms of photosynthesis, cell metabolism, and locomotive behaviors, such as gravitaxis and phototaxis (Richter et al. 2002; Daiker et al. 2010, 2011; Schwartzbach and Shigeoka 2017). Recent advances in experimental techniques, such as gene knockdown, have facilitated E. gracilis gene function studies. For example, a photoactivated adenylyl cyclase was reported to be required as a photoreceptor for phototaxis (Iseki et al. 2002). In contrast, it remains very difficult to obtain nuclear mutants of E. gracilis; few traits are induced by mutagens, such as ultraviolet light or ethyl methanesulfonate, which has hampered the complete inhibition of a specific gene functions, and is suggested to be related to the species' polyploidy (Schiff and Epstein 1965; Hill et al. 1966). Accordingly, few locomotion-defective mutant strains have been identified and characterized (Schiff et al. 1980). Although a chloroplast mutant of E. gracilis, $\mathrm{M}_{2}^{-} \mathrm{BUL}$, was previously reported to lack motility (Shneyour and Avron 1975), it has not been extensively examined; it is reported to lack photosynthesis activity and is not available in current culture collections, e.g., The Sammlung von Algenkulturen der Universität Göttingen (Culture Collection of Algae at Göttingen University [SAG], DEU), to which various E. gracilis wildtype strains are deposited.

In addition to its utilization as a model organism, E. gracilis has been exploited for industrial applications because of its fast proliferation, nutrient-rich features, and characteristic metabolism (Schwartzbach and Shigeoka 2017). As a saccharide reserve, E. gracilis stores paramylon, a crystalline form of $\beta$-(1,3)-D-glucan, which has various applications in the food industry (Watanabe et al. 2013; Russo et al. 2017). Thus, E. gracilis cells are particularly suitable as an ingredient in functional foods and supplements. Moreover, the stored paramylon is metabolized under hypoxic conditions into wax ester, which is suitable as a biofuel source (Inui et al. 1982, 1983). To enhance these industrial applications, E. gracilis has been bred to achieve thermostability and high oil production using Fe-ion irradiation as a mutagen (Yamada et al. $2016 a, b)$.

In the present study, we produced and characterized a non-motile mutant strain of $E$. gracilis $\left(\mathrm{M}_{3}^{-} \mathrm{ZFeL}\right)$. We hypothesized that the non-motile mutant strain has a higher paramylon production than the wild type, because it could save significant energy, which is mostly used for the swimming in the wild type (Hamilton et al. 1992; Tunçay et al. 2013). We used our previously established method, which uses Fe-ion beam irradiation as a mutagen agent, to produce non-motile mutants of E. gracilis (Yamada et al. 2016a, b). We screened for non-motile

Peer) reviewing PDF | (2020:04:47711:2:0:NEW 26 Aug 2020) 
117 cells from the Fe-ion beam-irradiated population and selected $\mathrm{M}^{-}{ }_{3} \mathrm{ZFeL}$ for further studies. The 118 proliferation rate, swimming and sedimentation speed, and paramylon and wax ester production 119 of $\mathrm{M}_{3}^{-} \mathrm{ZFeL}$ were measured. Our results demonstrated that Fe-ion beam irradiation and the 120 following screening method was an efficient strategy necessary to acquire non-motile E. gracilis

121

122

123

124

125

126

127

128

129

130

131

132

133

134

135

136

137

138

139

140

141

142

143

144

145

146

147

148

149

150

151

152

153

154

155

156 mutant strains, as well as the potential of the produced $\mathrm{M}_{3}^{-} \mathrm{ZFeL}$ strain for industrial application.

\section{Materials \& Methods}

Strains, culture, and media

The wild-type Z strain, which is identical to E. gracilis SAG $1224-5 / 25$, was provided by the culture collection of the Institute of Applied Microbiology (IAM), University of Tokyo, Tokyo, JPN. The $\mathrm{M}_{3}^{-} \mathrm{ZFeL}$ mutant strain produced in this study is deposited in the microbial culture collection at the National Institute for Environmental Studies (NIES, Tsukuba, JPN) with registration number NIES-4440. The cells were cultured using Cramer-Myers (CM) (Cramer and Myers 1952) and Koren-Hunter (KH) media (Koren and Hutner 1967) for autotrophic and heterotrophic growth, respectively, prepared at $\mathrm{pH}$ 3.5. The $\mathrm{CM}$ medium does not include a carbon source, while the KH medium includes $12 \mathrm{~g} \mathrm{~L}^{-1}$ of glucose and various organic acids and amino acids as carbon sources. The seed culture was maintained in KH medium with each month of subculture.

\section{Fe-ion irradiation and mutant screening}

The mutant strain was obtained through Fe-ion irradiation using the same conditions as previously reported (Yamada et al. 2016a, b), and mutant screening was based on the algal phenotypes. Two milliliters of an E. gracilis cell suspension $\left(4 \times 10^{5}\right.$ cells $\left.\mathrm{mL}^{-1}\right)$ was placed in hybridization bags that were segmented into $5 \times 7 \mathrm{~cm}$ compartments. They were then irradiated

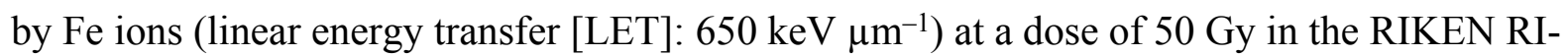
beam factory (Wako, Saitama, JPN). After one week of recovery by culture inoculation in KH culture medium, two rounds of mutant screening were conducted.

About $1 \times 10^{5}$ mutagenized cells with independent genetic backgrounds were placed at one end of a culture dish filled with $10 \mathrm{~mL}$ of liquid $\mathrm{KH}$ medium with $0.5 \%$ methyl cellulose, which was added to solidify and prevent unexpected stirring of the culture, and were illuminated from the opposite end with $50 \mu \mathrm{mol}$ photons $\mathrm{m}^{-2} \mathrm{~s}^{-1}$ of fluorescent light. After one week of culture incubation following Fe-ion beam irradiation, cells proliferated more than 100 times, resulting in $1 \times 10^{7}$ mutagenized cells, and most of them were found at the illuminated side through phototaxis. About 1,000 of the cells remained at the initial inoculation point; these were collected as putative non-motile mutant cells with a micropipette.

The collected population was supposed to include a high proportion of motile cells, which resided at the initial inoculation point due to random dispersal; therefore, they were subjected to a second round of screening. The cells were placed at the opening end of a $15 \mathrm{~mL}$ conical tube filled with $5 \mathrm{~mL}$ of liquid $\mathrm{KH}$ medium with $0.5 \%$ methyl cellulose, illuminated with 
$15750 \mu \mathrm{mol}$ photons $\mathrm{m}^{-2} \mathrm{~s}^{-1}$ of fluorescent light, and shaded with aluminum foil, except at the

158 closing end. After 2 weeks of static horizontal incubation in the tubes, the cells proliferated more

159 than 1,000 times. Most cells were gathered at the unshaded end to seek light. We retrieved about

1601,000 of the cells that showed no movement from the initial inoculation position and cultured

161 them for an additional two weeks.

162 The proliferated cells were randomly isolated using fluorescence-activated cell sorting 163 (MoFlo XDP; Beckman Coulter, Brea, CA, USA) to establish clonal lines in individual wells of

164

165

166

167

168

169

170

171

172

173

174

175

176

177

178

179

180

181

182

183

184

185

186

187

188

189

190

191

192

193

194

195

196 96-well plates (Tissue Culture Test Plate; TPP, Trasadingen, CHE) filled with $200 \mu \mathrm{L}$ of KH medium. Populations without cell motility were selected by microscopic observations at $26^{\circ} \mathrm{C}$ and identified as the motility-defective strains. The above-mentioned screening was performed for two independently mutagenized populations. During the screening process, cells proliferated more than $10^{6}$ times; therefore, each population included many genetically identical cells. Based on this possibility, one mutant strain was established from each population, i.e., two strains were established from the two independent populations.

\section{Morphological characterization}

The cells were observed under an upright light microscope (DM2500B; Leica, Wetzlar, DEU) equipped with a differential interference contrast module. Scanning electron microscopy (SEM) was conducted using a field emission scanning electron microscope (SU8200; Hitachi, Tokyo, JPN) after following a general sample preparation. Briefly, $1 \mathrm{~mL}$ of culture in $\mathrm{KH}$ medium was centrifuged at $2,000 \times \mathrm{g}$ for $1 \mathrm{~min}$ to collect the cells. The precipitated cells were pre-fixed by adding a solution containing $2.5 \%$ glutaraldehyde, $2 \%$ paraformaldehyde, and $0.1 \mathrm{M}$ sodium cacodylate, and fixed again using a solution containing $1 \%$ osmium tetroxide and $0.1 \mathrm{M}$ sodium cacodylate. The fixed samples were completely dried using a critical point dryer, sputter-coated with osmium, and then subjected to SEM photographing.

For quantifying the proportion of cells with flagella, the cells were cultured in $\mathrm{CM}$ or $\mathrm{KH}$ medium and used in their logarithmic growth phase for analysis. For the $\mathrm{CM}$ medium, the cells were cultured in $50 \mathrm{~mL}$ of medium using a $100 \mathrm{~mL}$ volume test tube aerated with $50 \mathrm{~mL}$ $\min ^{-1}$ of air containing $5 \% \mathrm{CO}_{2}$. For the $\mathrm{KH}$ medium, each strain was cultured in $50 \mathrm{~mL}$ of medium using a $100 \mathrm{~mL}$ volume conical flask with rotary shaking at $100 \mathrm{rpm}$. Each culture was conducted at $26^{\circ} \mathrm{C}$ with $100 \mu \mathrm{mol} \mathrm{m}{ }^{-2} \mathrm{~s}^{-1}$ of constant illumination and sub-cultured every week to maintain a stable proliferation. The cells were then fixed with glutaraldehyde $(0.025 \%)$ and observed for the presence of flagella under an inverted microscope (CKX41; Olympus, Tokyo, JPN) equipped with a phase contrast module. To exclude subjective judgment, short and intact flagella were not differentiated and were counted as cells with flagella.

\section{Growth tests}

The algae growth rate was evaluated in $100 \mathrm{~mL}$ test tubes containing $50 \mathrm{~mL}$ of medium. The cells were inoculated with an initial optical density (OD) of 0.1 and precultured for three days in $100 \mathrm{~mL}$ volume conical flasks containing $\mathrm{KH}$ medium with continuous shaking (100 rpm at 26 
197

198

199

200

201

202

203

204

205

206

207

208

209

210

211

212

213

214

215

216

217

218

219

220

221

222

223

224

225

226

227

228

229

230

231

232

233

234

235

236

${ }^{\circ} \mathrm{C}$, and $100 \mathrm{~m}^{-2} \mathrm{~s}^{-1}$ of constant illumination). The cultured cells were then collected by centrifuging at $2,000 \times \mathrm{g}$ for $5 \mathrm{~min}$ and washed twice with either $\mathrm{KH}$ or $\mathrm{CM}$ medium, which was subsequently used for the culture test. Next, the cell suspension was inoculated in culture tubes with an initial OD of 0.1 and incubated at $26{ }^{\circ} \mathrm{C}$ with $100 \mu \mathrm{mol} \mathrm{m}^{-2} \mathrm{~s}^{-1}$ of constant illumination. $\mathrm{KH}$ and $\mathrm{CM}$ cultures were aerated with $50 \mathrm{~mL} \mathrm{~min}^{-1}$ of air containing $5 \% \mathrm{CO}_{2}$. ODs from culture growth were determined over time by spectrophotometer (UVmini-1240; Shimadzu, Kyoto, JPN) at $\lambda 680 \mathrm{~nm}$, immediately after placing the suspension in the cuvette.

\section{Quantitative analysis of motility}

We used microscopic observations with video image capture to quantitatively evaluate algae motility. Observation and image processing techniques were used as previously reported (Ozasa et al. 2011). We confined the cells in suspension $(0.7 \mu \mathrm{L}$, containing 300-500 cells) in a closed circular polydimethylsiloxane (PDMS) micro-chamber (2.49 mm diameter and $140 \mu \mathrm{m}$ depth), and observed red-light, bright-field transmission images with a $5 \times$ objective lens at $26^{\circ} \mathrm{C}$. Approximately 300-500 cells were measured in the micro-chamber. Cell movements were visualized by differentiating, thresholding, and superimposing the video images sequentially (trace image), and evaluated by counting the spatial sum of the trace pixels in the trace image (Ozasa et al. 2013, 2014). The number of trace pixels was named the "trace momentum" (TM) (Ozasa et al. 2013; Ozasa et al. 2014). The measurement rate of the TM values was $0.67 \mathrm{~Hz}$ (one frame per $1.6 \mathrm{~s}$ ). The TM value is a fair measure of all locomotive activity observed within the chamber. The typical swimming traces were individually superimposed onto the cell distribution image and prepared as a binary image to demonstrate the cell's swimming activity (Ozasa et al. 2016).

\section{Sedimentation analysis}

Cellular sedimentation rates in $\mathrm{KH}$ and $\mathrm{CM}$ media at $26^{\circ} \mathrm{C}$ were evaluated in $1.5 \mathrm{~mL}$ microtubes. The culture was prepared as indicated above for the morphology analysis. In brief, when $\mathrm{CM}$ medium was used, cells were cultured in $100 \mathrm{~mL}$ volume test tubes with aeration, whereas with $\mathrm{KH}$ medium, they were cultured in a $100 \mathrm{~mL}$ volume conical flask with rotary shaking at $100 \mathrm{rpm}$. Each culture was conducted with constant light illumination $\left(100 \mu \mathrm{mol} \mathrm{m}^{-2}\right.$ $\mathrm{s}^{-1}$ ). Culture ODs average at $\lambda 680 \mathrm{~nm}$ were 23.4 and 22.0 by KH culture, and 4.1 and 4.4 by $\mathrm{CM}$ culture of wild-type and $\mathrm{M}_{3}^{-} \mathrm{ZFeL}$ strains, respectively. Images of the cell suspension in each microtubule were taken at $1-5 \mathrm{~min}$ intervals with a general compact digital camera. The transparent supernatant area was detected and quantified from the images using the Image $\mathrm{J}$ software, with the threshold values set as 0 to 50 after converting to greyscale. Identical rectangular regions were selected inside the images of microtube images, and the transparent area inside each region was quantified.

To evaluate the sedimentation speed at $20 \mathrm{~cm}$ depth, the E. gracilis wild type and the $\mathrm{M}^{-}$ ${ }_{3} \mathrm{ZFeL}$ mutant strains were cultured in cuboid acryl beakers $(10 \times 10 \times 30 \mathrm{~cm})$ using $2 \mathrm{~L}$ of CM medium at $29{ }^{\circ} \mathrm{C}$ and $1,300 \mu \mathrm{mol} \mathrm{m}^{-2} \mathrm{~s}^{-1}$ of overhead illumination (light-dark ratio of $12: 12 \mathrm{~h}$ ). 
237 Beakers were filled with approximately the same concentrations $\left(\mathrm{g} \mathrm{L}^{-1}\right)$ of cells, 0.37 and $0.32 \mathrm{~g}$ $238 \mathrm{~L}^{-1}$ for the wild-type and $\mathrm{M}^{-} 3 \mathrm{ZFeL}$ strains, respectively. After stirring the culture, sedimentation

239

240

241

242

243

244

245

246

247

248

249

250

251

252

253

254

255

256

257

258

259

260

261

262

263

264

265

266

267

268

269

270

271

272

273

274 275 was periodically observed for $3.5 \mathrm{~h}$, and supernatant was carefully removed to obtain a concentrated culture. Sediment concentration was then quantified by weighing the dried cells after filtration with a glass fiber filter (GA-55; ADVANTEC, Tokyo, JPN).

\section{Quantification of carbohydrates and lipids}

The harvested algal cells were freeze-dried (FDV-1200; EYELA, Tokyo, JPN), and the deproteinized paramylon and lipid components were extracted as previously reported (Inui et al. 1982; Suzuki et al. 2015). The dried cells (10 mg) were sonicated twice in $10 \mathrm{~mL}$ of acetone for $90 \mathrm{~s}$ (UD-201; TOMY, Tokyo, JPN) with dial setting at 4 in $50 \mathrm{~mL}$ volume of plastic centrifuge tubes. The paramylon was subsequently separated from the residual components using centrifugation, boiled for $30 \mathrm{~min}$ in $10 \mathrm{~mL}$ of $1 \%$ sodium dodecyl sulfate aqueous solution, and washed twice with $10 \mathrm{~mL}$ of water. The extracted paramylon was then quantified using the phenol-sulfuric acid method (Montgomery 1957), which can quantify total carbohydrates. Similarly, neutral lipids were extracted from $100 \mathrm{mg}$ of dried cells using n-hexane as a solvent; $10 \mathrm{~mL}$ of $\mathrm{n}$-hexane was added to the dried cells in $50 \mathrm{~mL}$ glass centrifuge tubes. The suspension was then homogenized for $90 \mathrm{~s}$ using a sonicator (UD-201; TOMY, Tokyo, JPN) with dial setting at 4 , and then filtered with a piece of glass fiber filter paper $(\mathrm{GF} / \mathrm{C}$; Whatman, Little Chalfont, Buckinghamshire, UK), followed by an additional step of residue extraction. After evaporating the collected organic solvent dissolving lipids, the weight of the residue left in the flask was quantified as that of the extracted total neutral lipid.

\section{Statistical analyses}

The results with error bars, except that of motility quantification, are represented as mean \pm standard error from three independent experiments. The results of motility quantification are represented as mean \pm standard deviation (SD) for the whole measured time points. Statistical significance was analyzed using Student's $t$-test. For multiple comparisons, Bonferroni's corrections were applied. $p<0.05$ was considered significant.

\section{Results}

\section{Characterization of $\mathrm{M}_{3}^{-} \mathrm{ZFeL}$}

Through our screening for non-motile mutants of E. gracilis, we established two mutants with independent genetic backgrounds. The mutant strains were named $\mathrm{M}_{3}^{-} \mathrm{ZFeL}$ and $\mathrm{M}_{4}^{-} \mathrm{ZFeL}$ according to the typical nomenclature (Schiff et al. 1980), with the phenotypic designation "M" for motility and the mutagen designation "Fe" for Fe-ion irradiation (Yamada et al. 2016a). The strain $\mathrm{M}_{3}^{-} \mathrm{ZFeL}$ showed a stable behavioral phenotype, and was thus used in this study. 
276

277

278

279

280

281

282

283

284

285

286

287

288

289

290

291

292

293

294

295

296

297

298

299

300

301

302

303

304

305

306

307

308

309

310

311

312

313

314

315

The E. gracilis $\mathrm{M}^{-}{ }_{3} \mathrm{ZFeL}$ strain showed non-motile phenotype with few defects in proliferation. This phenotype was identified by the formation of colonies from single-cell inoculation in liquid culture. The $\mathrm{M}_{3}^{-} \mathrm{ZFeL}$ strain showed colony formation in liquid $\mathrm{KH}$ culture medium after a week, whereas wild-type cells were dispersed in the same culture medium (Fig. $1 \mathrm{~A}$ and $\mathrm{B})$. As judged by both light and electron microscopy, the $\mathrm{M}_{3}^{-} \mathrm{ZFeL}$ cells were more rounded than were the wild-type cells. In addition, the $\mathrm{M}^{-}{ }_{3} \mathrm{ZFeL}$ flagella were shorter than those of the wild type (Fig. 1C-F). Moreover, a significantly higher proportion of $\mathrm{M}^{-}{ }_{3} \mathrm{ZFeL}$ cells lacked flagella both in the $\mathrm{CM}\left(p=5.6 \times 10^{-5}, t(4)=15.1\right)$ and $\mathrm{KH}(p=0.04, t(4)=2.29)$ media (Fig. $1 \mathrm{G}$ and $\mathrm{H}$ ).

The autotrophic and heterotrophic growth rates of the wild-type and $\mathrm{M}_{3}^{-} \mathrm{ZFeL}$ strains were evaluated using $\mathrm{KH}$ and CM culture media (Fig. 2). The mutant strain showed slightly slower and faster growth than did the wild type in the $\mathrm{KH}$ and $\mathrm{CM}$ culture, respectively. The $\mathrm{M}^{-}{ }_{3} \mathrm{ZFeL}$ culture ODs at the third $(p=0.020, t(4)=-5.81)$ and fourth $\left(p=1.5 \times 10^{-4}, t(4)=-20.48\right)$ days of $\mathrm{KH}$ culture, which included high amounts of glucose, were significantly lower than those of the wild type (Fig. 2A). On the other hand, the ODs on the second $(p=0.020, t(4)=-6.13)$, fourth $(p=0.042, t(4)=-4.98)$, and eleventh $(p=0.036, t(4)=-5.18)$ days of CM culture were significantly higher than those of the wild type (Fig. 2B).

Our quantification of motility showed that the $\mathrm{M}_{3}^{-} \mathrm{ZFeL}$ TM values were less than onethousandth that of the wild type. Following previous studies, cells cultured in a liquid $\mathrm{KH}$ medium were adequately stirred and subjected to TM measurements. Fig. $3 \mathrm{~A}$ shows the cell distribution and swimming traces over approximately $8 \mathrm{~s}$. The absence of swimming traces for the mutant strain revealed that cells could not move using their flagella. The total TM value $\pm S D$ for the wild-type strain was $11,161 \pm 269$ for 368 cells, whereas that for $\mathrm{M}^{-}{ }_{3} \mathrm{ZFeL}$ was only $9 \pm$ 6 for 467 cells, as shown in Fig. 3B. The TM value for the mutant strain cannot be distinguished from the measurement noise, indicating that the $\mathrm{M}^{-}{ }_{3} \mathrm{ZFeL}$ strain was almost completely nonmotile. Although some of the $\mathrm{M}^{-} \mathrm{ZFeL}$ cells possessed a short flagellum (Fig. $1 \mathrm{C}-\mathrm{H}$ ), our results indicated that the short flagellum did not function well.

\section{Potential of $\mathrm{M}_{3}^{-} \mathrm{ZFeL}$ for industrial application}

$\mathrm{M}^{-}{ }_{3} \mathrm{ZFeL}$ demonstrated faster sedimentation than did the wild type in $1.5 \mathrm{~mL}$ microtubes. As shown in Fig. 4, the $\mathrm{M}_{3}^{-} \mathrm{ZFeL}$ cell sedimentation rate was higher than that of the wild-type cells under both heterotrophic (Fig. 4A) and autotrophic (Fig. 4B) growth conditions using KH and $\mathrm{CM}$ media, respectively. The values at the final time point $(40 \mathrm{~min})$ were significantly different under both heterotrophic $(p=0.010, t(4)=-3.69)$ and autotrophic $(p=0.011, t(4)=-3.60)$ conditions (Fig. 4A and B).

By analyzing sedimentation in a larger-scale culture, $\mathrm{M}_{3}^{-} \mathrm{ZFeL}$ was found to be advantageous for industrial harvesting. To assess the effect of fast sedimentation on the harvest 
316 of cells cultured using photosynthesis, wild-type and $\mathrm{M}_{3}^{-} \mathrm{ZFeL}$ strains were cultured using $\mathrm{CM}$ 317 medium and their sedimentation rates were measured in $20 \mathrm{~cm}$-deep beakers. $\mathrm{M}_{3}^{-} \mathrm{ZFeL}$ cells 318 showed a significantly faster sedimentation rate than did the wild-type cells (Fig. 5). The $\mathrm{M}^{-}$ $319{ }_{3} \mathrm{ZFeL}$ cell density at the bottom of the beaker $\left(34.1 \mathrm{~g} \mathrm{~L}^{-1}\right)$, was more than 10 times that of the 320 wild type $\left(3.0 \mathrm{~g} \mathrm{~L}^{-1}\right)$.

321

322

323

324

325

326

327

328

329

330

331

332

333

334

335

336

337

338

339

340

341

342

343

344

345

346

347

348

349

350

351

352

353

354

The paramylon storage in $\mathrm{M}_{3}^{-} \mathrm{ZFeL}$ cells was equal to or greater than that in the wild-type cells, whereas lipid accumulation in $\mathrm{M}^{-}{ }_{3} \mathrm{ZFeL}$ cells was less than (autotrophic) or equal to (heterotrophic) that that in the wild-type cells. As shown in Fig. $6 \mathrm{~A}$ and $\mathrm{B}, \mathrm{M}^{-}{ }_{3} \mathrm{ZFeL}$ cells produced a higher amount of paramylon (1.6 times; $p=0.033, t(4)=-2.51)$ than did the wildtype cells under autotrophic conditions, whereas there was no significant difference under heterotrophic conditions. In contrast, the $\mathrm{M}^{-}{ }_{3} \mathrm{ZFeL}$ strain showed a lower lipid content than did the wild type, especially under autotrophic conditions $(p=0.034, t(4)=-2.47)$. (Fig. $6 \mathrm{C}$ and D).

\section{Discussion}

The non-motile and fast sedimentation phenotypes of the $\mathrm{M}_{3}^{-} \mathrm{ZFeL}$ mutant strain were considered to have strong correlations with the loss of negative gravitaxis. Wild-type E. gracilis exhibited positive and negative gravitaxis that depended on its environmental and cellular status (Stallwitz and Häder 1994; Lebert et al. 1999). Recent research has shown that wild-type $E$. gracilis uses mechano-sensing proteins and flagellar beating to stay at a preferential water depth (Häder and Hemmersbach 2017), rather than using buoyancy control. Since $\mathrm{M}^{-}{ }_{3} \mathrm{ZFeL}$ cells were incapable of flagellar beating, they could not use negative gravitaxis to prevent sinking. In addition, the highly accumulated paramylon, which had a particle density of approximately 1.5 in $\mathrm{M}^{-} \mathrm{ZFeL}$ cells, may also contribute to the increase in their specific gravity and sinking under autotrophic conditions. Our sedimentation tests showed that the faster sedimentation of the $\mathrm{M}^{-}$ 3ZFeL cells was achieved not only in small microtubes but also inside a deeper reservoir, thus being advantageous for industrial harvesting. Typically, cells in heterotrophic cultures sediment faster than those in autotrophic cultures, probably due to the higher carbohydrate accumulation. However, our results in microtubes showed that both strains sedimented faster in the autotrophic culture than in the heterotrophic culture. This seems to be due to the differences in cell density of the cultures; stacking highly concentrated cells at the bottom of the KH culture inhibited further sedimentation of the cells.

Flagellar motion is derived from the driving force of the doublet microtubules and dynein in the flagellum. Defects in the dynein, axonemal proteins, or related components result in a lack of flagellar movement (Turner 2006; Wang et al. 2014). We have not yet identified the component, or more specifically, the gene, responsible for the immobility of the $\mathrm{M}^{-}{ }_{3} \mathrm{ZFeL}$ mutant strain. However, the lack of motility possibly reduces the cellular energy consumption and glycolysis. Such suppression is reported to result in enhanced starch accumulation in 
355

356

357

358

359

360

361

362

363

364

365

366

367

368

369

370

371

372

373

374

375

376

377

378

379

380

381

382

383

384

385

386

387

388

389

390

391

392

393

394

Chlamydomonas (Hamilton et al. 1992; Tunçay et al. 2013), which may explain why greater paramylon storage was observed in the $\mathrm{M}_{3}^{-} \mathrm{ZFeL}$ cells.

The $\mathrm{M}_{3}^{-} \mathrm{ZFeL}$ strain showed slightly slower growth in the heterotrophic $\mathrm{KH}$ culture than did the wild type, suggesting that the function of glucose metabolism was slightly disturbed in the mutant. Meanwhile, the $\mathrm{M}^{-}{ }_{3} \mathrm{ZFeL}$ strain showed faster growth in the autotrophic $\mathrm{CM}$ culture, which did not include carbon sources, suggesting that the mutant strain had an intact photosynthesis system and consumed the photosynthesized resources slower than the wild type. Moreover, the $\mathrm{M}_{3}^{-} \mathrm{ZFeL}$ had no defect in paramylon production, thus indicating that $\mathrm{M}^{-}{ }_{3} \mathrm{ZFeL}$ may improve industrial paramylon production. Although a limited defect was observed in the accumulation of lipids, $\mathrm{M}_{3}^{-} \mathrm{ZFeL}$ would also be applicable to lipid production. The higher accumulation of paramylon in $\mathrm{M}^{-}{ }_{3} \mathrm{ZFeL}$ may be partially due to the energy conservation by not swimming. However, since the proliferation speed of $\mathrm{M}_{3}^{-} \mathrm{ZFeL}$ was not drastically faster than the wild type, even in the autotrophic culture, the amount of energy conservation in $\mathrm{M}^{-}{ }_{3} \mathrm{ZFeL}$ does not seem significant.

The $\mathrm{M}^{-}{ }_{3} \mathrm{ZFeL}$ characterization results suggest that the mutant is defective in the components related to flagellum motion and/or formation that are not critical for survival and proliferation, at least under controlled laboratory conditions. The production of mutant strains by mutagenesis using high-LET irradiations, such as Ar-ion or Fe-ion irradiation, is advantageous because it causes a small number of large deletions in the genome with few side mutations (Hirano et al. 2015; Kazama et al. 2017). Although we could not identify the genetic cause of the phenotype in $\mathrm{M}_{3}^{-} \mathrm{ZFeL}$, the process of breeding these strains ensures minimal side mutations. This will enable the immediate industrial use of the strain by reducing the possibility of showing unexpected and undesirable traits, which may not be observed in the laboratory but appear under harsh and unstable outdoor-culture conditions.

The $\mathrm{M}_{3}^{-} \mathrm{ZFeL}$ mutant of $E$. gracilis produced in this study is highly promising for the industrial production of food ingredients and chemical substances because it evidenced fast sedimentation, high paramylon storage, and a growth speed comparable to that of the wild type, at least under autotrophic conditions. In particular, the fast sedimentation of the mutant strain will save time and energy during the harvesting processes, which will contribute to the use of $E$. gracilis as a feedstock for biofuel by improving the balance between the energy invested to produce it and the energy output. In practical cultivation to produce paramylon and lipid under autotrophic conditions, processes to accumulate respective ingredients are added. In particular, cultured cells are subjected to nitrogen-restricted conditions to accumulate paramylon; the culture is then condensed and hypoxically conditioned to ferment the paramylon to wax ester (Suzuki 2017). Our results showed that $\mathrm{M}^{-}{ }_{3} \mathrm{ZFeL}$ can competently accumulate larger amounts of paramylon under heterotrophic culture conditions than under autotrophic conditions; therefore, we suggest that they also show sufficient paramylon accumulation by nitrogen restriction (Briand 
395

396

397

398

399

400

401

402

403

404

405

406

407

408

409

410

411

412

413

414

415

416

417

418

419

420

421

422

423

424

425

426

427

428

429

430

431

432

433

434

and Calvayrac 1980; Sumida et al. 1987), which enables the subsequent accumulation of wax ester (Inui et al. 2017). In contrast, the fast-sinking trait of the cells may cause sedimentation in industrial-scale culture ponds during cultivation. To test this and improve the mass-cultivation method, further experiments using practical outdoor-culture ponds are required.

In addition to the production cost, non-motile cells have the advantage of being easy to observe and manipulate in a limited area under the microscope. This will be helpful for further basic studies on E. gracilis, which should include a long-lasting evaluation of a specific cell, e.g., observing its basic physiological phenomena, such as cell division (Figure S1 and Supplemental movie), as well as the micro-manipulation of single cells, such as that through single-cell electroporation, to deliver genes and proteins to the cells (Ohmachi et al. 2016).

\section{Conclusions}

This study demonstrated the successful production of a motility-defective mutant of the microalga $E$. gracilis, which has been industrially used in recent years. The acquired mutant showed amoebic motility but no swimming using the flagellum, and accumulated more carbohydrates than did the wild type under autotrophic culture conditions. However, the growth results indicated that the energy conservation due to the non-motile phenotype was not significant. Instead, owing to the inability to migrate and the accumulation of carbohydrates with high specific gravity, the cells sedimented faster during incubation. This trait indicates the potential to save energy for harvesting by introducing a natural sedimentation process. Therefore, the produced motility-defective mutant of E. gracilis and its derivatives may be useful for the industrial production of E. gracilis.

\section{Acknowledgments}

The heavy-ion beam irradiation was conducted at the RI beam factory (RIBF), which is operated by the RIKEN Nishina Center and Center for Nuclear Study, University of Tokyo. The SEM images of the cells were obtained with the assistance of Dr. Toyooka (RIKEN, CSRS, JPN). We would like to thank Editage (www.editage.com) for English language editing.

\section{References}

Bovee EC (1982) Movement and locomotion of Euglena. In: The Biology of EUGLENA VolumeIII: Physiology. Academic Press, New York., pp 143-168

Briand J, Calvayrac R (1980) Paramylon synthesis in heterotrophic and photoheterotrophic Euglena (euglenophyceae). J Phycol 16:234-239

Cramer M, Myers J (1952) Growth and photosynthetic characteristics of Euglena gracilis. $17: 384-402$ 
435

436

437

438

439

440

441

442

443

444

445

446

447

448

449

450

451

452

453

454

455

456

457

458

459

460

461

462

463

464

465

466

467

468

469

470

471

472

473

474

475

476

Daiker V, Häder D-P, Richter PR, Lebert M (2011) The involvement of a protein kinase in phototaxis and gravitaxis of Euglena gracilis. Planta 233:1055-1062. doi: 10.1007/s00425011-1364-5

Daiker V, Lebert M, Richter P, Häder D-P (2010) Molecular characterization of a calmodulin involved in the signal transduction chain of gravitaxis in Euglena gracilis. Planta 231:12291236. doi: 10.1007/s00425-010-1126-9

Dusenbery, DB (2009) Living at micro scale: the unexpected physics of being small. Harvard University Press ( Cambridge, MA); ISBN: 978-0-674-03116-6.

Domenici P, Claireaux G, McKenzie DJ (2007) Environmental constraints upon locomotion and predator-prey interactions in aquatic organisms: an introduction. Philos Trans R Soc Lond B Biol Sci 362:1929-36 . doi: 10.1098/rstb.2007.2078

Dusenbery DB (1997) Minimum size limit for useful locomotion by free-swimming microbes. Proc Natl Acad Sci U S A 94:10949-54. doi: 10.1073/pnas.94.20.10949

Häder D-P, Hemmersbach R (2017) Gravitaxis in Euglena. Adv Exp Med Biol 979:237-266. doi: 10.1007/978-3-319-54910-1_12

Hamilton BS, Nakamura K, Roncari DA (1992) Accumulation of starch in Chlamydomonas reinhardtii flagellar mutants. Biochem Cell Biol 70:255-8

Harris EH (2001) Chlamydomonas as a model organism. Annu Rev Plant Physiol Plant Mol Biol 52:363-406. doi: 10.1146/annurev.arplant.52.1.363

Hill HZ, Schiff J, Epstein HT (1966) Studies of chloroplast development in Euglena. Biophys J 6:373-383. doi: 10.1038/185825a0

Hirano T, Kazama Y, Ishii K, Ohbu S, Shirakawa Y, Abe T (2015) Comprehensive identification of mutations induced by heavy-ion beam irradiation in Arabidopsis thaliana. Plant J 82:93104. doi: 10.1111/tpj.12793

Inui H, Ishikawa T, Tamoi M (2017) Wax Ester Fermentation and Its Application for Biofuel Production. Adv Exp Med Biol 979:269-283. doi: 10.1007/978-3-319-54910-1_13

Inui H, Miyatake K, Nakano Y, Kitaoka S (1983) Production and composition of wax esters by fermentation of Euglena gracilis. Agric Biol Chem 47:2669-2671. doi: 10.1080/00021369.1983.10866013

Inui H, Miyatake K, Nakano Y, Kitaoka S (1982) Wax ester fermentation in Euglena gracilis. FEBS Lett 150:89-93. doi: 10.1016/0014-5793(82)81310-0

Iseki M, Matsunaga S, Murakami A, Ohno K, Shiga K, Yoshida K, Sugai M, Takahashi T, Hori T, Watanabe M (2002) A blue-light-activated adenylyl cyclase mediates photoavoidance in Euglena gracilis. Nature 415:1047-1051. doi: 10.1038/4151047a

Jahn TL, Bovee EC (1965) Movement and locomotion of microorganisms. Annu Rev Microbiol 19:21-58. doi: 10.1146/annurev.mi.19.100165.000321

Jékely G, Colombelli J, Hausen H, Guy K, Stelzer E, Nédélec F, Arendt D (2008) Mechanism of phototaxis in marine zooplankton. Nature 456:395-399. doi: 10.1038/nature07590

Kamiya R (1991) Selection of Chlamydomonas dynein mutants. Methods Enzymol 196:348-55. doi: 10.1016/0076-6879(91)96031-1

Kamiya R (1995) Exploring the function of inner and outer dynein arms with Chlamydomonas mutants. Cell Motil Cytoskeleton 32:98-102. doi: 10.1002/cm.970320205

Peer) reviewing PDF | (2020:04:47711:2:0:NEW 26 Aug 2020) 
477 Kamiya R, Kurimoto E, Muto E (1991) Two types of Chlamydomonas flagellar mutants missing

478

479

480

481

482

483

484

485

486

487

488

489

490

491

492

493

494

495

496

497

498

499

500

501

502

503

504

505

506

507

508

509

510

511

512

513

514

515

516

517

518 different components of inner-arm dynein. J Cell Biol 112:441-7. doi: $10.1083 / \mathrm{jcb} .112 .3 .441$

Kamiya R, Okamoto M (1985) A mutant of Chlamydomonas reinhardtii that lacks the flagellar outer dynein arm but can swim. J Cell Sci 74:181-91

Kazama Y, Ishii K, Hirano T, Wakana T, Yamada M, Ohbu S, Abe T (2017) Different mutational function of low- and high-linear energy transfer heavy-ion irradiation demonstrated by whole-genome resequencing of Arabidopsis mutants. Plant J 92:10201030. doi: 10.1111/tpj.13738

Koren LE, Hutner SH (1967) High-yield media for photosynthesizing Euglena gracilis Z. J Protozool 14:(Suppl.) 17

Miyata M, Robinson RC, Uyeda TQP, Fukumori Y, Fukushima S-I, Haruta S, Homma M, Inaba K, Ito M, Kaito C, Kato K, Kenri T, Kinosita Y, Kojima S, Minamino T, Mori H, Nakamura S, Nakane D, Nakayama K, Nishiyama M, Shibata S, Shimabukuro K, Tamakoshi M, Taoka A, Tashiro Y, Tulum I, Wada H, Wakabayashi K-I (2020) Tree of motility - A proposed history of motility systems in the tree of life. Genes Cells 25:6-21. doi: $10.1111 /$ gtc. 12737

Lebert M, Porst M, Richter P, Hader DP (1999) Physical characterization of gravitaxis in Euglena gracilis. J Plant Physiol 155:338-43. doi: 10.1016/s0176-1617(99)80114-X

Montgomery R (1957) Determination of glycogen. Arch Biochem Biophys 67:378-386. doi: 10.1016/0003-9861(57)90292-8

Ohmachi M, Fujiwara Y, Muramatsu S, Yamada K, Iwata O, Suzuki K, Wang DO (2016) A modified single-cell electroporation method for molecule delivery into a motile protist, Euglena gracilis. J Microbiol Methods 130:106-111. doi: 10.1016/j.mimet.2016.08.018

Okita N (2005) Phototactic activity in Chlamydomonas "non-phototactic" mutants deficient in $\mathrm{Ca} 2+$-dependent control of flagellar dominance or in inner-arm dynein. J Cell Sci 118:529537. doi: 10.1242/jcs.01633

Ozasa K, Lee J, Song S, Hara M, Maeda M (2011) Two-dimensional optical feedback control of Euglena confined in closed-type microfluidic channels. Lab Chip 11:1933. doi: 10.1039/c01c00719f

Ozasa K, Lee J, Song S, Hara M, Maeda M (2013) Gas/liquid sensing via chemotaxis of Euglena cells confined in an isolated micro-aquarium. Lab Chip 13:4033. doi: 10.1039/c31c50696g

Ozasa K, Lee J, Song S, Maeda M (2014) Transient freezing behavior in photophobic responses of Euglena gracilis investigated in a microfluidic device. Plant Cell Physiol 55:1704-1712. doi: 10.1093/pcp/pcu101

Ozasa K, Won J, Song S, Maeda M (2016) Autonomous oscillation/separation of cell density artificially induced by optical interlink feedback as designed interaction between two isolated microalgae chips. Sci Rep 6:24602. doi: 10.1038/srep24602

Richter P, Ntefidou M, Streb C, Lebert M, Hader D-P (2002) Physiological characterization of gravitaxis in Euglena gracilis. J Gravit Physiol 9: 279-280

Roberts AM (2010) The mechanics of gravitaxis in Paramecium. J Exp Biol 213:4158-4162. doi: $10.1242 /$ jeb.050666 
519 Roberts AM (2006) Mechanisms of gravitaxis in Chlamydomonas. Biol Bull 210:78-80. doi: $520 \quad 10.2307 / 4134597$

521 Russo R, Barsanti L, Evangelista V, Frassanito AM, Longo V, Pucci L, Penno G, Gualtieri P 522 (2017) Euglena gracilis paramylon activates human lymphocytes by upregulating pro523 inflammatory factors. Food Sci Nutr 5:205-214. doi: 10.1002/fsn3.383

524 Schiff JA, Epstein HT (1965) The continuity of the chloroplast in Euglena. In: Locke M (ed)

525 Reproduction: Molecular, subcellular, and cellular. Academic Press, New York, pp 131$526 \quad 189$

527

528

529

530

531

532

533

534

535

536

537

538

539

540

541

542

543

544

545

546

547

548

549

550

551

552

553

554

555

556

557

558

Schiff JA, Lyman H, Russell GK (1980) [2] Isolation of mutants of Euglena gracilis: An addenum. Methods Enzymol 69:23-29. doi: 10.1016/S0076-6879(80)69004-1

Schwartzbach SD, Shigeoka S (eds) (2017) Euglena: Biochemistry, cell and molecular biology. Springer International Publishing, Cham

Shneyour A, Avron M (1975) Properties of photosynthetic mutants isolated from Euglena gracilis. Plant Physiol 55:137-141

Sourjik V, Wingreen NS (2012) Responding to chemical gradients: bacterial chemotaxis. Curr Opin Cell Biol 24:262-268. doi: 10.1016/j.ceb.2011.11.008

Stallwitz E, Hader DP (1994) Effects of heavy metals on motility and gravitactic orientation of the flagellate, Euglena gracilis. Eur J Protistol 30:18-24. doi: 10.1016/s09324739(11)80194-x

Sumida S, Ehara T, Osafune T, Hase E (1987) Ammonia- and light-induced degradation of paramylum in Euglena gracilis. Plant Cell Physiol 28:1587-1592

Suzuki K (2017) Large-Scale Cultivation of Euglena. Adv Exp Med Biol 979:285-293. doi: 10.1007/978-3-319-54910-1_14

Suzuki K, Mitra S, Iwata O, Ishikawa T, Kato S, Yamada K (2015) Selection and characterization of Euglena anabaena var. minor as a new candidate Euglena species for industrial application. Biosci Biotechnol Biochem 79:. doi: $10.1080 / 09168451.2015 .1045828$

Tunçay H, Findinier J, Duchêne T, Cogez V, Cousin C, Peltier G, Ball SG, Dauvillée D (2013) A forward genetic approach in Chlamydomonas reinhardtii as a strategy for exploring starch catabolism. PLoS One 8:e74763. doi: 10.1371/journal.pone.0074763

Turner RM (2006) Moving to the beat: a review of mammalian sperm motility regulation. Reprod Fertil Dev 18:25. doi: 10.1071/RD05120

Wang H, Gau B, Slade WO, Juergens M, Li P, Hicks LM (2014) The Global Phosphoproteome of Chlamydomonas reinhardtii reveals complex organellar phosphorylation in the flagella and thylakoid membrane. Mol Cell Proteomics 13:2337-2353. doi: 10.1074/mcp.M114.038281

Watanabe T, Shimada R, Matsuyama A, Yuasa M, Sawamura H, Yoshida E, Suzuki K (2013) Antitumor activity of the $\beta$-glucan paramylon from Euglena against preneoplastic colonic aberrant crypt foci in mice. Food Funct 4:1685-1690. doi: 10.1039/c3fo60256g

Webre DJ, Wolanin PM, Stock JB (2003) Bacterial chemotaxis. Curr Biol 13:R47-9

Peer) reviewing PDF | (2020:04:47711:2:0:NEW 26 Aug 2020) 
559 Yamada K, Kazama Y, Mitra S, Marukawa Y, Arashida R, Abe T, Ishikawa T, Suzuki K (2016a)

560 Production of a thermal stress resistant mutant Euglena gracilis strain using Fe-ion beam

561 irradiation. Biosci Biotechnol Biochem 8451:1-7. doi: 10.1080/09168451.2016.1171702

562 Yamada K, Suzuki H, Takeuchi T, Kazama Y, Mitra S, Abe T, Goda K, Suzuki K, Iwata O

563 (2016b) Efficient selective breeding of live oil-rich Euglena gracilis with fluorescence-

564 activated cell sorting. Sci Rep 6:26327. doi: 10.1038/srep26327

565

566 


\section{Figure 1}

Fig. 1. $\mathrm{M}_{3}^{-} \mathrm{ZFeL}$ and wild-type strains of Euglena gracilis in static culture

( $A$ and $B$ ) Photographs of wild-type (A) and $\mathrm{M}_{3}^{-} \mathrm{ZFeL}(\mathrm{B})$ colonies grown in Koren-Hunter $(\mathrm{KH})$ medium on a 96-well plate for one week. (C-F) Microphotographs ( $C$ and D) and scanning electron micrographs ( $E$ and $F$ ) of wild-type ( $C$ and $E$ ) and $M_{3}^{-} Z F e L(D$ and $F$ ) cells cultured in $100 \mathrm{~mL}$ volume conical flasks containing $\mathrm{KH}$ medium with continuous shaking (100 rpm, 26 ${ }^{\circ} \mathrm{C}$, and $100 \mu \mathrm{mol} \mathrm{m} \mathrm{m}^{-2} \mathrm{~s}^{-1}$ of constant illumination). Scale bars indicate $20 \mu \mathrm{m}$. (G and $\mathrm{H}$ ) The proportion of cells that possess flagella among the wild-type and $\mathrm{M}_{3}^{-} \mathrm{ZFeL}$ cells in the Cramer-Myers (CM) $(\mathrm{G})$ and $\mathrm{KH}$ media $(\mathrm{H})$. More than 200 cells were observed for each condition in one day. The observations were conducted three times on different days. Error bars show the standard errors for three replicates. $* p<0.05$, Student's $t$-test. 
(A)

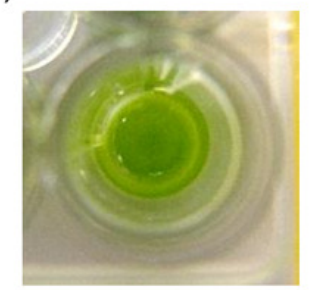

(E)

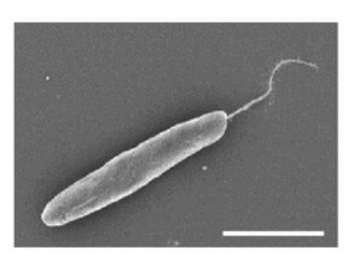

(B)

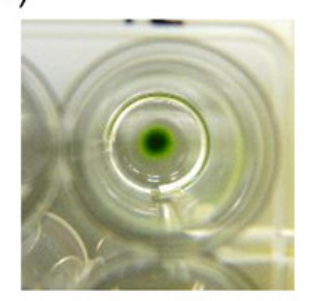

(F)

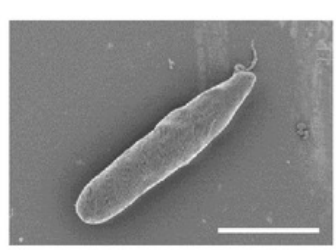

(C)

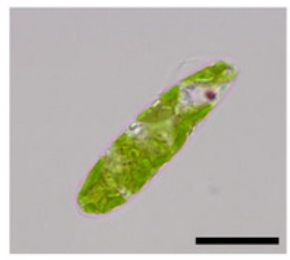

(G)

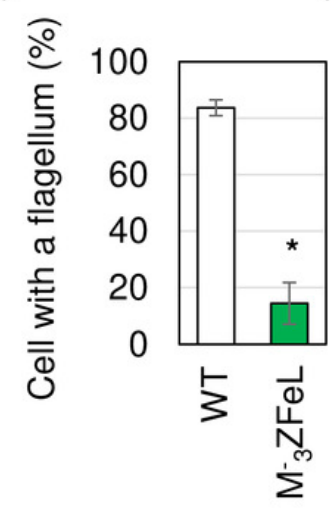

(D)

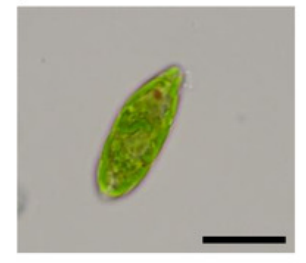

(H)

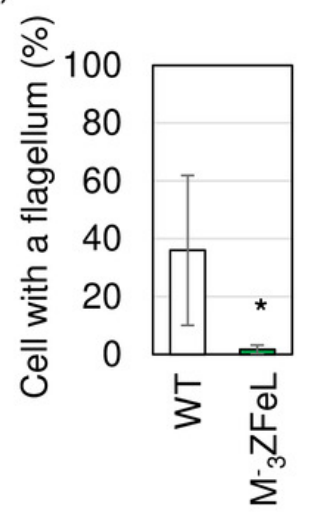




\section{Figure 2}

Fig. 2. Growth curves of $\mathrm{M}_{3}^{-} \mathrm{ZFeL}$ and wild-type strains of Euglena gracilis

( $A$ and $B$ ) Growth curves of the wild-type (white) and $\mathrm{M}_{3}^{-} \mathrm{ZFeL}$ (green) strains on heterotrophic $\mathrm{KH}(\mathrm{A})$ and autotrophic CM (B) media. The initial culture was inoculated into a medium with an optical density of 0.1 measured at $\lambda 680 \mathrm{~nm}$. The growth was determined on days $0,1,2$, $3,4,6,7,8$, and 9 after the start of the Koren-Hunter $(\mathrm{KH})$ culture, while it was determined on days $0,1,2,4,5,7,8,10,11,14$, and 15 after the start of the Cramer-Myers (CM) culture. The growth test was conducted in triplicate using three independent tubes. The error bars indicate the standard errors for these triplicates. ${ }^{*} p<0.05$, Student's $t$-test with Bonferroni's correction. 
(A)

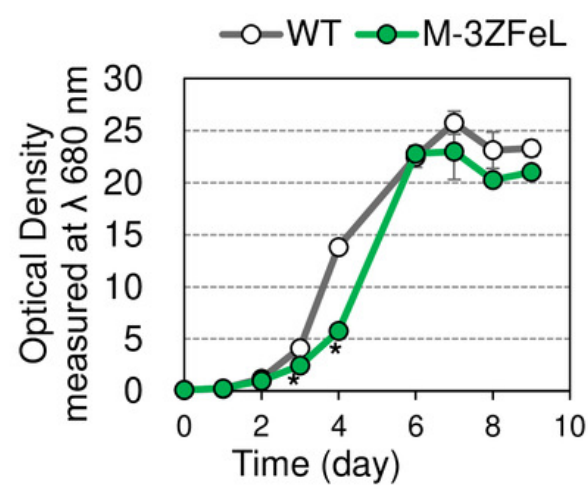

(B)

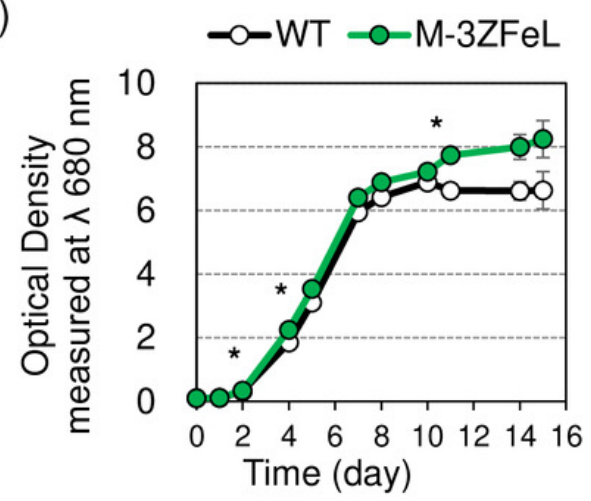




\section{Figure 3}

Fig. 3. Motility quantification of $\mathrm{M}_{3}^{-} \mathrm{ZFeL}$ and wild-type strains of Euglena gracilis using trace momentum (TM)

(A) Cell distribution and swimming traces over approximately $8 \mathrm{~s}$ for the wild-type (left panel) and $\mathrm{M}_{3}^{-} \mathrm{ZFeL}$ (right panel) strains. Both cell types were grown in heterotrophic $(\mathrm{KH})$ medium and measured during the logarithmic growth phase. The magnified images of the areas within the squares are shown in the lower left of the panels. The diameter of these circles are approximately $2.5 \mathrm{~mm}$. Green dots and light green lines indicate $E$. gracilis cells and their traces, respectively. (B) The motility of the wild-type and $\mathrm{M}_{3}^{-} \mathrm{ZFeL}$ strains, evaluated using TM. The TM was obtained every $1.6 \mathrm{~s}$ and averaged over $10 \mathrm{~min}$. Error bars show the standard deviation for 500 time points. 
(A)

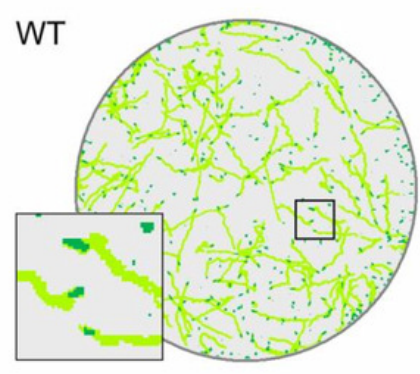

(B)

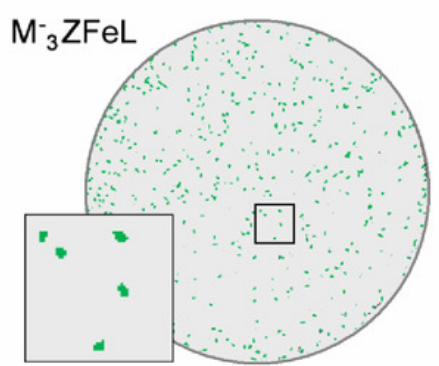

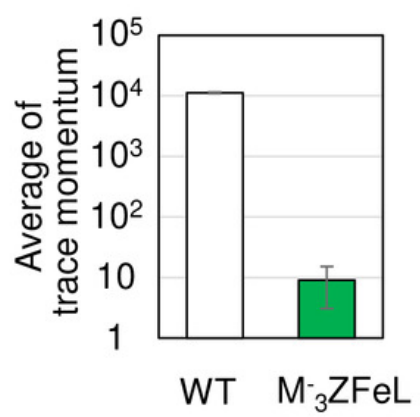




\section{Figure 4}

Fig. 4. Sedimentation speed of $\mathrm{M}_{3}^{-} \mathrm{ZFeL}$ and wild-type strains of Euglena gracilis

( $\mathrm{A}$ and $\mathrm{B}$ ) Sedimentation rates of the wild type (white) and $\mathrm{M}_{3}^{-} \mathrm{ZFeL}$ (green) in Koren-Hunter (KH) (heterotrophic; A) and Cramer-Myers (CM) (autotrophic; B) media. Photographs of the cell suspension were taken every 1 min to measure the transparent area in each $1.5 \mathrm{~mL}$ tube. After cell sedimentation, the supernatant becomes transparent, and the area on the photograph was used to evaluate the sedimentation rate. Error bars show standard errors for three replicates. a.u., arbitrary unit. 
(A)

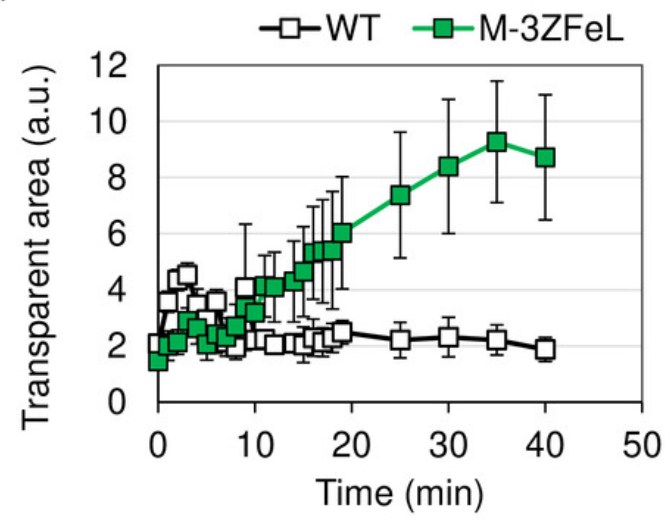

(B)

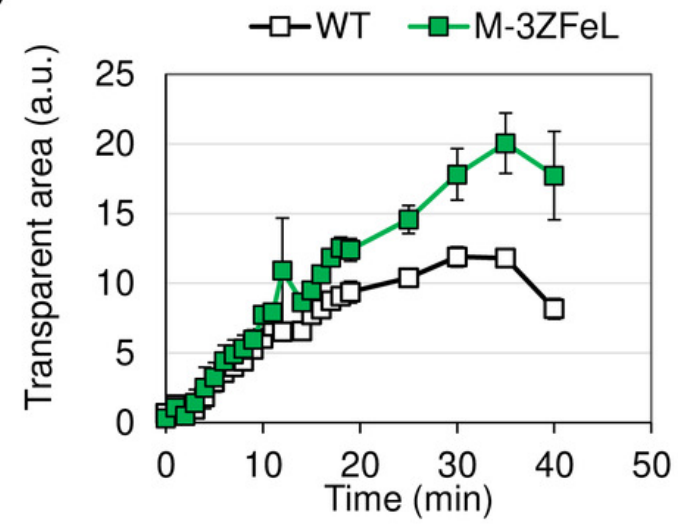




\section{Figure 5}

Fig. 5. Sedimentation rate of Euglena gracilis cells in $20 \mathrm{~cm}$-deep beakers

(A) Autotrophic cultures of the wild-type (left) and $\mathrm{M}_{3}^{-} \mathrm{ZFeL}$ (right) strains suspended in $2 \mathrm{~L}$ beakers and left to stand under static conditions for $3.5 \mathrm{~h}$. Cramer-Myers (CM) medium was used for the culture. (B) The sediment before and after $3.5 \mathrm{~h}$ of sedimentation. The cells that were retrieved from the bottom of the beaker and quantified for their dry weight are indicated with an arrowhead in (A). 
(A)

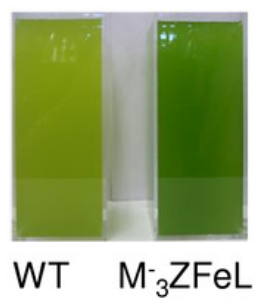

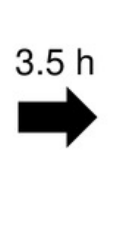

WT $\quad \mathrm{M}_{3} \mathrm{ZFeL}$
(B)

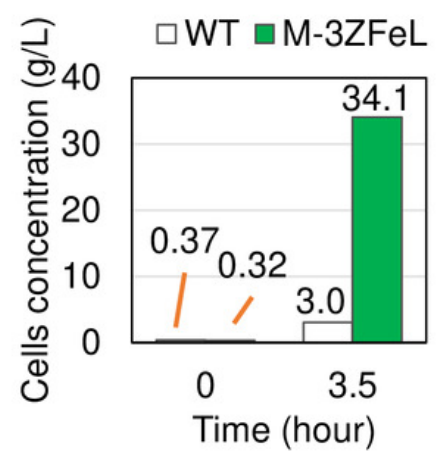




\section{Figure 6}

Fig. 6. Paramylon storage and lipid content of $\mathrm{M}_{3}^{-} \mathrm{ZFeL}$ and wild-type strains of Euglena gracilis cells

(A-D) Paramylon storage ( $A$ and $B$ ) and lipid content ( $C$ and $D$ ) in the wild-type (white) and

$\mathrm{M}_{3}^{-} \mathrm{ZFeL}$ (green) strains cultured in heterotrophic ( $\mathrm{A}$ and $\mathrm{C}$ ) and autotrophic ( $\mathrm{B}$ and $\mathrm{D}$ ) media.

Paramylon and lipid were extracted and quantified from freeze-dried cells, and their percentage weights were calculated. Cells were collected during their logarithmic growth phase. The $y$-axis scale is different in each figure. Error bars show standard errors for three replicates. ${ }^{*} p<0.05$, Student's $t$-test. 
(A)

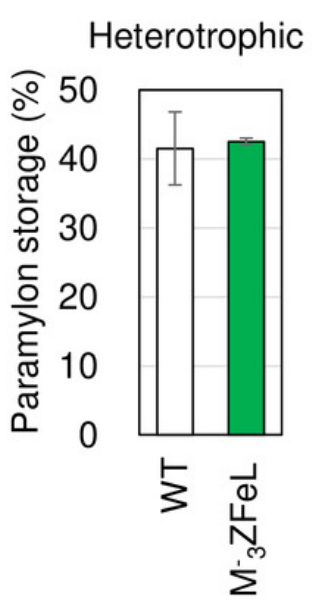

(B)

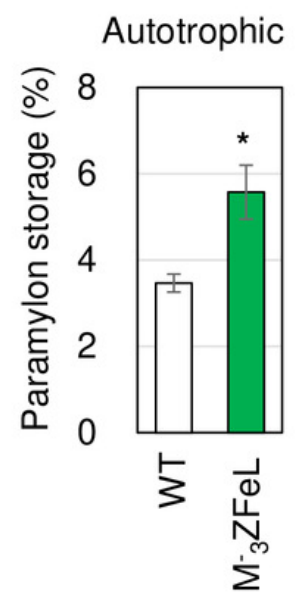

(C)

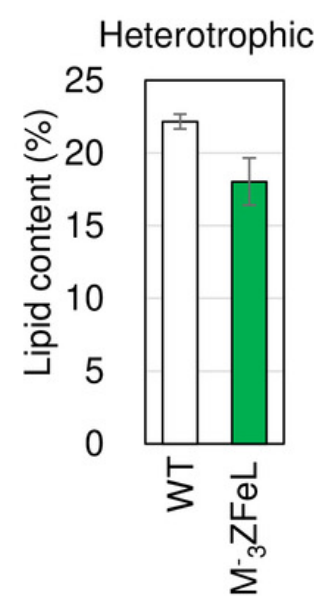

(D)

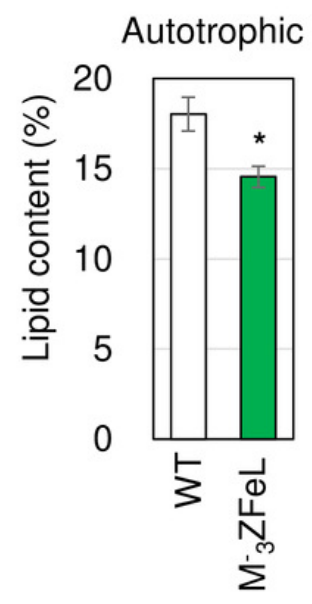

